\title{
Article
}

\section{A unified integral operator and further its consequences}

\author{
Ghulam Farid \\ COMSATS University Islamabad, Attock Campus, Pakistan.; ghlmfarid@ciit-attock.edu.pk
}

Received: 21 December 2019; Accepted: 15 January 2020; Published: 9 February 2020.

\begin{abstract}
The aim of this paper is to construct left sided and right sided integral operators in a unified form. These integral operators produce various well known integral operators in the theory of fractional calculus. Formulated integral operators of this study include generalized fractional integral operators of Riemann-Liouville type and operators containing Mittag-Leffler functions in their kernels. Also boundedness of all these fractional integral operators is derived from the boundedness of unified integral operators. The existence of new integral operators may have useful consequences in applied sciences besides in fractional calculus.
\end{abstract}

Keywords: Integral operators, fractional integral operators, bounds.

MSC: 26D10, 31A10, 26A33, 33E12.

\section{Introduction}

e start with the following compact form of fractional integrals:

Definition 1. [1] Let $f:[a, b] \rightarrow \mathbb{R}$ be an integrable function. Also let $g$ be an increasing and positive function on $(a, b]$, having a continuous derivative $g^{\prime}$ on $(a, b)$. The left-sided and right-sided fractional integrals of a function $f$ with respect to another function $g$ on $[a, b]$ of order $\mu \in \mathbb{C}(\mathcal{R}(\mu)>0)$ are defined as:

$$
{ }_{g}^{\mu} I_{a^{+}} f(x)=\frac{1}{\Gamma(\mu)} \int_{a}^{x}(g(x)-g(t))^{\mu-1} g^{\prime}(t) f(t) d t, x>a
$$

and

$$
{ }_{g}^{\mu} I_{b^{-}} f(x)=\frac{1}{\Gamma(\mu)} \int_{x}^{b}(g(t)-g(x))^{\mu-1} g^{\prime}(t) f(t) d t, x<b,
$$

where $\Gamma($.$) is the gamma function.$

A $k$-fractional analogue of above definition is given as follows:

Definition 2. [2] Let $f:[a, b] \rightarrow \mathbb{R}$ be an integrable function. Also let $g$ be an increasing and positive function on $(a, b]$, having a continuous derivative $g^{\prime}$ on $(a, b)$. The left-sided and right-sided $k$-fractional integral operators, $k>0$ of a function $f$ with respect to another function $g$ on $[a, b]$ of order $\mu \in \mathbb{C}(\mathcal{R}(\mu)>0)$ are defined as:

$$
{ }_{g}^{\mu} I_{a^{+}}^{k} f(x)=\frac{1}{k \Gamma_{k}(\mu)} \int_{a}^{x}(g(x)-g(t))^{\frac{\mu}{k}-1} g^{\prime}(t) f(t) d t, x>a
$$

and

$$
{ }_{g}^{\mu} I_{b^{-}}^{k} f(x)=\frac{1}{k \Gamma_{k}(\mu)} \int_{x}^{b}(g(t)-g(x))^{\frac{\mu}{k}-1} g^{\prime}(t) f(t) d t, x<b,
$$

where

$$
\Gamma_{k}(\mu)=\int_{0}^{\infty} t^{\mu-1} e^{\frac{-t^{k}}{k}} d t
$$

is the $k$-gamma function. 
Fractional integral operators which have been studied in recent decades are special cases of generalized classical integral operators of Riemann-Liouville type which are defined in (1) and (2). Instead of studying the derivations of recently defined fractional integral operators from (3) and (4), the authors of these decades have derived them independently, see [1,3-8]. Remark 1 provides derivations of these fractional integrals from (1) and (2) and along with their $k$-analogues (3) and (4). A detail study of fractional integrals associated with (3) and (4), which have been investigated by the authors of recent years, is summarized in the following remark:

Remark 1. Fractional integrals elaborated in (3) and (4) particularly produce several known fractional integrals corresponding to different settings of $k$ and $g$.

1. For $k=1$ (3) and (4) fractional integrals coincide with (1) and (2) fractional integrals.

2. By taking $g$ as identity function (3) and (4) fractional integrals coincide with $k$-fractional Riemann-Liouville integrals defined by Mubeen et al. in [7].

3. For $k=1$, along with $g$ as identity function (3) and (4) fractional integrals coincide with Riemann-Liouville fractional integrals [1].

4. For $k=1$ and $g(x)=\frac{x^{\rho}}{\rho}, \rho>0$, (3) and (4) produce Katugampola fractional integrals defined by Chen $e t$ al. in [3].

5. For $k=1$ and $g(x)=\frac{x^{\tau+s}}{\tau+s}$, (3) and (4) produce generalized conformable fractional integrals defined by Khan et al. in [6].

6. If we take $g(x)=\frac{(x-a)^{s}}{s}, s>0$ in (3) and $g(x)=-\frac{(b-x)^{s}}{s}, s>0$ in (4), then conformable $(k, s)$-fractional integrals are achieved as defined by Habib et al. in [4].

7. If we take $g(x)=\frac{x^{1+s}}{1+s}$, then conformable fractional integrals are achieved as defined by Sarikaya et al. in [8].

8. If we take $g(x)=\frac{(x-a)^{s}}{s}, s>0$ in (3) and $g(x)=-\frac{(b-x)^{s}}{s}, s>0$ in (4) with $k=1$, then conformable fractional integrals are achieved as defined by Jarad et al. in [5].

Moreover, the authors also have constructed various fractional integral operators by using Mittag-Leffler function and its generalizations. Recently, Andrić et al. studied an extended generalized Mittag-Leffler function and the associated fractional integral operators in [9]. The extended generalized fractional integrals defined in [9], produce all fractional integrals containing Mittag-Leffler function defined in [10-13].

Definition 3. [9] Let $\omega, \mu, \alpha, l, \gamma, c \in C, \Re(\mu), \Re(\alpha), \Re(l)>0, \Re(c)>\Re(\gamma)>0$ with $p \geq 0, \delta>0$ and $0<v \leq \delta+\Re(\mu)$. Let $f \in L_{1}[a, b]$ and $x \in[a, b]$. Then the generalized fractional integral operators $\epsilon_{\mu, \alpha, l, \omega, a^{+}}^{\gamma, \delta, v, c} f$ and $\epsilon_{\mu, \alpha, l, \omega, b^{-}}^{\gamma, \delta, v, c} f$ are defined by:

$$
\left(\epsilon_{\mu, \alpha, l, \omega, a^{+}}^{\gamma, \delta, v, c} f\right)(x ; p)=\int_{a}^{x}(x-t)^{\alpha-1} E_{\mu, \alpha, l}^{\gamma, \delta, v, c}\left(\omega(x-t)^{\mu} ; p\right) f(t) d t
$$

and

$$
\left(\epsilon_{\mu, \alpha, l, \omega, b^{-}}^{\gamma, \delta, v, c} f\right)(x ; p)=\int_{x}^{b}(t-x)^{\alpha-1} E_{\mu, \alpha, l}^{\gamma, \delta, v, c}\left(\omega(t-x)^{\mu} ; p\right) f(t) d t
$$

where

$$
E_{\mu, \alpha, l}^{\gamma, \delta, v, c}(t ; p)=\sum_{n=0}^{\infty} \frac{\beta_{p}(\gamma+n v, c-\gamma)}{\beta(\gamma, c-\gamma)} \frac{(c)_{n v}}{\Gamma(\mu n+\alpha)} \frac{t^{n}}{(l)_{n \delta}}
$$

is the extended generalized Mittag-Leffler function,

$$
\beta_{p}(x, y)=\int_{0}^{1} t^{x-1}(1-t)^{y-1} e^{-\frac{p}{t(1-t)}} d t
$$

and $(c)_{n v}=\frac{\Gamma(c+n v)}{\Gamma(c)}$.

Remark 2. The settings of $\omega, v, \delta, l, p, \gamma$ into generalized Mittag-Leffler function obtain the following consequences:

1. Setting $p=0,(5)$ and (6) reduce to the fractional integral operators defined by Salim-Faraj in [10]. 
2. Setting $l=\delta=1,(5)$ and (6) reduce to the fractional integral operators defined by Rahman et al. in [11].

3. Setting $p=0$ and $l=\delta=1,(5)$ and (6) reduce to the fractional integral operators defined by Shukla-Prajpati in [12].

4. Setting $p=0$ and $l=\delta=v=1$, (5) and (6) reduce to the fractional integral operators defined by Prabhakar in [13].

5. Setting $p=\omega=0$, (5) and (6) reduce to the left-sided and right-sided Riemann-Liouville fractional integrals.

The extended generalized Mittag-Leffler function $E_{\mu, \alpha, l}^{\gamma, \delta, v, c}(t ; p)$ is absolutely convergent for $v<\delta+\Re(\mu)$, (see [9]). If $S$ is the sum of series of absolute terms of the Mittag-Leffler function $E_{\mu, \alpha, l}^{\gamma, \delta, v, c}(t ; p)$, then one can obtain $\left|E_{\mu, \alpha, l}^{\gamma, \delta, c}(t ; p)\right| \leq S$. This property of absolutely convergence of the Mittag-Leffler function is used in establishing Theorems 6, 7, 8. The rest of the paper is organized as follows: In Section 2, we establish the existence of integral operators in a unified form. The bounds of these unified integral operators are also obtained. It is important to note that the unified integral operators produce almost all Riemann-Liouville type fractional integral operators as well as fractional integral operators containing the Mittag-Leffler function in their kernels. Furthermore, the bounds of all these fractional integral operators are obtained in Section 2 and Section 3, from the bounds which have been established for unified integral operators.

\section{Existence of new unified integral operators}

The first result provides the existence of new integral operators with upper bounds in variable form.

Theorem 4. Let $f:[a, b] \longrightarrow \mathbb{R}, 0<a<b$, be a positive and integrable function, $g:[a, b] \longrightarrow \mathbb{R}$ be differentiable and strictly increasing. Also let $\frac{\phi}{x}$ be an increasing function on $[a, b]$ and $\omega, \alpha, l, \gamma, c \in \mathbb{C}, \Re(\alpha), \Re(l)>0, \Re(c)>\Re(\gamma)>$ 0 with $p \geq 0, \mu, \delta>0$ and $0<v \leq \delta+\mu$. Then for $x \in[a, b]$, we have

$$
\int_{a}^{x} \frac{\phi(g(x)-g(t))}{g(x)-g(t)} E_{\mu, \alpha, l,}^{\gamma, \delta, v, c}\left(\omega(g(x)-g(t))^{\mu} ; p\right) g^{\prime}(t) f(t) d t \leq \phi(g(x)-g(a)) E_{\mu, \alpha, l,}^{\gamma, \delta, v, c}\left(\omega(g(x)-g(a))^{\mu} ; p\right)\|f\|_{[a, x]}
$$

and

$$
\int_{x}^{b} \frac{\phi(g(t)-g(x))}{g(t)-g(x)} E_{\mu, \alpha, l,}^{\gamma, \delta, v, c}\left(\omega(g(t)-g(x))^{\mu} ; p\right) g^{\prime}(t) f(t) d t \leq \phi(g(b)-g(x)) E_{\mu, \alpha, l,}^{\gamma, \delta, c}\left(w(g(b)-g(x))^{\mu} ; p\right)\|f\|_{[x, b]}
$$

where $\|f\|_{[a, x]}=\sup _{t \in[a, x]}|f(t)|$ and $\|f\|_{[x, b]}=\sup _{t \in[x, b]}|f(t)|$.

Proof. As $g$ is increasing, therefore for $t \in[a, x) ; x \in[a, b], g(x)-g(t) \leq g(x)-g(a)$. The function $\frac{\phi}{x}$ is increasing, therefore one can obtain:

$$
\frac{\phi(g(x)-g(t))}{g(x)-g(t)} \leq \frac{\phi(g(x)-g(a))}{g(x)-g(a)}
$$

It is given that $f$ is positive and $g$ is differentiable and increasing. Therefore from (9), the following inequality is valid:

$$
\frac{\phi(g(x)-g(t))}{g(x)-g(t)} g^{\prime}(t) f(t) \leq \frac{\phi(g(x)-g(a))}{g(x)-g(a)} g^{\prime}(t) f(t)
$$

Multiplying (10) by $E_{\mu, \alpha, l,}^{\gamma, \delta, c}\left(\omega(g(x)-g(t))^{\mu} ; p\right)$, then integrating over $[a, x]$, we obtain

$$
\begin{aligned}
& \int_{a}^{x} \frac{\phi(g(x)-g(t))}{g(x)-g(t)} E_{\mu, \alpha, l}^{\gamma, \delta, v, c}\left(\omega(g(x)-g(t))^{\mu} ; p\right) g^{\prime}(t) f(t) d t \\
& \leq \frac{\phi(g(x)-g(a))}{g(x)-g(a)} \int_{a}^{x} E_{\mu, \alpha, l}^{\gamma, \delta, v, c}\left(\omega(g(x)-g(t))^{\mu} ; p\right) g^{\prime}(t) f(t) d t
\end{aligned}
$$




$$
\begin{aligned}
& \int_{a}^{x} \frac{\phi(g(x)-g(t))}{g(x)-g(t)} E_{\mu, \alpha, l}^{\gamma, \delta, v, c}\left(\omega(g(x)-g(t))^{\mu} ; p\right) g^{\prime}(t) f(t) d t \\
& \leq \frac{\phi(g(x)-g(a))}{g(x)-g(a)} \sum_{n=0}^{\infty} \frac{\beta_{p}(\gamma+n v, c-\gamma)}{\beta(\gamma, c-\gamma)} \frac{(c)_{n v}}{\Gamma(\mu n+\alpha)(l)_{n \delta}}\|f\|_{[a, x]} \int_{a}^{x} \omega^{n}(g(x)-g(t))^{\mu n} g^{\prime}(t) d t .
\end{aligned}
$$

From which by solving integral and using the fact $\frac{1}{\mu n+1} \leq 1$, inequality (8) can be obtained.

Now on the other hand for $t \in(x, b]$ and $x \in[a, b]$, the following inequality holds true:

$$
\frac{\phi(g(t)-g(x))}{(g(t)-g(x))} g^{\prime}(t) f(t) \leq \frac{\phi(g(b)-g(x))}{(g(b)-g(x))} g^{\prime}(t) f(t) .
$$

Multiplying (13) by $E_{\mu, \alpha, l}^{\gamma, \delta, v, c}\left(\omega(g(t)-g(x))^{\mu} ; p\right)$, then integrating over $(x, b]$, we obtain

$$
\begin{aligned}
& \int_{x}^{b} \frac{\phi(g(t)-g(x))}{g(t)-g(x)} E_{\mu, \alpha, l}^{\gamma, \delta, v, c}\left(\omega(g(t)-g(x))^{\mu} ; p\right) g^{\prime}(t) f(t) d t \\
& \leq \frac{\phi(g(b)-g(x))}{g(b)-g(x)} \int_{x}^{b} E_{\mu, \alpha, l}^{\gamma, \delta, v, c}\left(\omega(g(t)-g(x))^{\mu} ; p\right) g^{\prime}(t) f(t) d t .
\end{aligned}
$$

From which the inequality (9) can be obtained.

From above theorem, we are motivated to give the definition of a new unified two-sided integral operator as follows:

Definition 5. Let $f, g:[a, b] \longrightarrow \mathbb{R}, 0<a<b$, be the functions such that $f$ be positive and $f \in L_{1}[a, b]$, and $g$ be differentiable and strictly increasing. Also let $\frac{\phi}{x}$ be an increasing function on $[a, \infty)$ and $\omega, \alpha, l, \gamma, c \in \mathbb{C}$, $\Re(\alpha), \Re(l)>0, \Re(c)>\Re(\gamma)>0$ with $p \geq 0, \mu, \delta>0$ and $0<v \leq \delta+\mu$. Then for $x \in[a, b]$ the left and right integral operators are defined by

$$
\left({ }_{g} F_{\mu, \alpha, l, a^{+}}^{\phi, \gamma, \delta, v, c} f\right)(x ; p)=\int_{a}^{x} \frac{\phi(g(x)-g(t))}{g(x)-g(t)} E_{\mu, \alpha, l}^{\gamma, \delta, v}\left(\omega(g(x)-g(t))^{\mu} ; p\right) g^{\prime}(t) f(t) d t
$$

and

$$
\left({ }_{g} F_{\mu, \alpha, l, b, b^{-}}^{\phi, \gamma} f\right)(x ; p)=\int_{x}^{b} \frac{\phi(g(t)-g(x))}{g(t)-g(x)} E_{\mu, \alpha, l}^{\gamma, \delta, v, c}\left(\omega(g(t)-g(x))^{\mu} ; p\right) g^{\prime}(t) f(t) d t .
$$

The upcoming theorem provides the boundedness of the unified integral operators.

Theorem 6. Under the assumption of Theorem 4, the following bounds hold for integral operators (15) and (16):

$$
\left|\left({ }_{g} F_{\mu, \alpha, l, a^{+}}^{\phi, \gamma} \delta\right)(x ; p)\right| \leq S|\phi(g(b)-g(a))|\|f\|_{[a, b]}
$$

and

Hence

$$
\left|\left({ }_{g} F_{\mu, \alpha, l, l, b^{-}}^{\phi, \gamma, \delta} f\right)(x ; p)\right| \leq S|\phi(g(b)-g(a))|\|f\|_{[a, b]} .
$$

$$
\left|\left({ }_{g} F_{\mu, \alpha, l, l, a^{+}}^{\phi, \gamma, c} f\right)(x ; p)+\left({ }_{g} F_{\mu, \alpha, l, l, b^{-}}^{\phi, \gamma, \delta, v, c} f\right)(x ; p)\right| \leq 2 S|\phi(g(b)-g(a))|\|f\|_{[a, b]},
$$

where $S$ is the sum of absolute terms of series in (7) and $\|f\|_{[a, b]}=\sup _{t \in[a, b]}|f(t)|$.

Proof. From (11), it can be obtained

$$
\left|\int_{a}^{x} \frac{\phi(g(x)-g(t))}{g(x)-g(t)} E_{\mu, \alpha, l}^{\gamma, \delta, c, c}\left(\omega(g(x)-g(t))^{\mu} ; p\right) g^{\prime}(t) f(t) d t\right| \leq S\left|\frac{\phi(g(x)-g(a))}{g(x)-g(a)}\right| \int_{a}^{x}\left|g^{\prime}(t) f(t)\right| d t
$$


by simplifying the above inequality we get

$$
\mid\left(g_{\mu, \alpha, l, a^{+}}^{\phi, \gamma, \delta, v} f(x ; p)|\leq S| \frac{\phi(g(x)-g(a))}{g(x)-g(a)} \mid\|f\|_{[a, x]}(g(x)-g(a)) .\right.
$$

As $g(x)-g(a) \leq g(b)-g(a)$, therefore

$$
\frac{\phi(g(x)-g(a))}{g(x)-g(a)} \leq \frac{\phi(g(b)-g(a))}{g(b)-g(a)}
$$

and hence (17) can be achieved.

Similarly from (14), one can obtain

$$
\left|\left({ }_{g} F_{\mu, \alpha, l, b, b^{-}}^{\phi, r, \delta} f\right)(x ; p)\right| \leq S\left|\frac{\phi(g(b)-g(x))}{g(b)-g(x)}\right|\|f\|_{(x, b]}(g(b)-g(x))
$$

and hence (18) can be achieved.

By using (17) and (18), the inequality in (19) can be obtained.

Integral operators defined in (15) and (16) have connection with fractional integral operators given in Section 1. The upcoming section describes the connection of these integral operators with fractional integral operators (5) and (6) and their bounds are computed.

\section{Bounds of fractional integral operators containing Mittag-Leffler functions}

In this whole section, we set the function $\phi(x)=x^{\alpha}, \alpha>0$ and the function $g(x)=I(x)$, where $I$ denotes the identity function. In this case bounds of fractional integral operators containing Mittag-Leffler functions defined in $[1,3-8]$ can be obtained at once from bounds of unified integral operators (15) and (16). As an example the bounds of fractional integral operators defined by Andrić et al. in [9] are obtained. Computation of rest of the bounds of related fractional integrals described in Remark 2 are left for the reader.

Theorem 7. The fractional integral operators of function $f$ defined in (5) and (6) are bounded for $\alpha>1$, further the following inequality holds:

$$
\left|\left(\epsilon_{\mu, \alpha, l, \omega, a^{+}}^{\gamma, \delta, v, c} f\right)(x ; p)+\left(\epsilon_{\mu, \alpha, l, \omega, b^{-}}^{\gamma, \delta, v, c} f\right)(x ; p)\right| \leq 2 S\left|b^{\alpha}-a^{\alpha}\right|\|f\|_{[a, b], \alpha}>1 .
$$

Proof. Let $g(x)=x$ and $\phi(x)=x^{\alpha}$. Then $\frac{\phi}{x}$ is increasing for $\alpha>1$. Therefore

$$
\left({ }_{g} F_{\mu, \alpha, l, l, a^{+}}^{\phi, \gamma, \delta, v, c} f\right)(x ; p)=\left({ }_{I} F_{\mu, \alpha, l, a^{+}}^{x^{\alpha}, \gamma, \delta, v, c} f\right)(x ; p):=\left(\epsilon_{\mu, \alpha, l, \omega, a^{+}}^{\gamma, \delta, v, c} f\right)(x ; p)
$$

and

$$
\left({ }_{g} F_{\mu, \alpha, l, b^{-}}^{\phi, \gamma, \delta, v, c} f\right)(x ; p)=\left({ }_{I} F_{\mu, \alpha, l, b^{-}}^{x^{\alpha}, \gamma, \delta, c} f\right)(x ; p):=\left(\epsilon_{\mu, \alpha, l, \omega, b^{-}}^{\gamma, \delta, v, c} f\right)(x ; p) .
$$

Thus

$$
\left|\left(\epsilon_{\mu, \alpha, l, \omega, a^{+}}^{\gamma, \delta, v, c} f\right)(x ; p)\right| \leq S\left|b^{\alpha}-a^{\alpha}\right|\|f\|_{[a, b]}
$$

and

$$
\left|\left(\epsilon_{\mu, \alpha, l, \omega, b^{-}}^{\gamma, \delta, v, c} f\right)(x ; p)\right| \leq S\left|b^{\alpha}-a^{\alpha}\right|\|f\|_{[a, b]} .
$$

Hence boundedness of fractional operators (5) and (6) is followed and (21) can be achieved.

Remark 3. By using (22) and (23), the boundedness of all fractional integrals containing Mittag-Leffler functions compiled in Remark 2 can be obtained. 


\section{Bounds of fractional integral operators associated with generalized $k$-fractional integrals}

In this whole section, we set the function $\phi(x)=\frac{x^{\frac{\beta}{k}}}{k \Gamma_{k}(\beta)}, \beta, k>0$. In this case bounds of fractional integral operators defined in [10-13] can be obtained at once from bounds of unified integral operators (15) and (16). As an example the bounds of fractional integral operators (3) and (4) are obtained. Computation of the rest of the bounds of related fractional integrals described in Remark 1 are left for the reader.

Theorem 8. The generalized fractional integral operators of function $f$ defined in (3) and (4) are bounded for $\beta>k$, further the following inequality holds:

$$
\left|g_{g}^{\beta} I_{a^{+}}^{k} f(x)+{ }_{g}^{\beta} I_{b^{-}}^{k} f(x)\right| \leq \frac{2 S}{k \Gamma_{k}(\beta)}\left|g(b)^{\beta}-g(a)^{\beta}\right|\|f\|_{[a, b]}, \beta>k .
$$

Proof. Let $\phi(x)=\frac{x^{\frac{\beta}{k}}}{k \Gamma_{k}(\beta)}, \beta, k>0$ and $p=\omega=0$. Then $\frac{\phi}{x}$ is increasing for $\beta>k$. Therefore

$$
\left({ }_{g} F_{\mu, \alpha, \alpha, l, a^{+}}^{\phi, \gamma, \delta} f\right)(x ; p)=\left({ }_{g} F_{\mu, \alpha, l, a^{+}}^{\frac{x_{k}^{k}}{k K_{k}(\beta)}, \gamma, \delta, v, c} f\right)(x ; 0):={ }_{g}^{\beta} I_{a^{+}}^{k} f(x)
$$

and

$$
\left({ }_{g} F_{\mu, \alpha, l, b^{-}}^{\phi, \gamma, \delta, v, c} f\right)(x ; p)=\left({ }_{g} F_{\mu, \alpha, l, b^{-}}^{\frac{x^{k}}{k \Gamma_{k}(\beta)}, \gamma, \delta, v, c} f\right)(x ; 0):={ }_{g}^{\beta} I_{b^{-}}^{k} f(x) .
$$

Thus

$$
\left|{ }_{g}^{\beta} I_{a^{+}}^{k} f(x)\right| \leq \frac{S}{k \Gamma_{k}(\beta)}\left|g(b)^{\beta}-g(a)^{\beta}\right|\|f\|_{[a, b]}
$$

and

$$
\left|{ }_{g}^{\beta} I_{b^{-}}^{k} f(x)\right| \leq \frac{S}{k \Gamma_{k}(\beta)}\left|g(b)^{\beta}-g(a)^{\beta}\right|\|f\|_{[a, b]} .
$$

Hence boundedness of fractional operators (3) and (4) is followed and (24) can be achieved.

Remark 4. By using (25) and (26), the boundedness of all fractional integrals compiled in Remark 1 can be obtained. Also by setting $g(x)=I(x)$ in (22) and (23) integral operators defined in [14] can be obtained and using Theorem 6 bounds of these integral operators can be achieved.

\section{Concluding remarks}

The aim of this study is to develop unified integral operators which provide the fractional integral operators of Riemann-Liouville type as well as fractional integral operators containing Mittag-Leffler functions in their kernels. The bounds of these new integral operators are computed which simultaneously provide the bounds of all fractional integral operators defined in [1,3-8,10-13]. The existence of new generalized integral operators may be useful in applied sciences along with the theory and applications of fractional calculus.

Author Contributions: All authors contributed equally to the writing of this paper. All authors read and approved the final manuscript.

Conflicts of Interest: "The authors declare no conflict of interest."

\section{References}

[1] Kilbas, A. A. A., Srivastava, H. M., \& Trujillo, J. J. (2006). Theory and applications of fractional differential equations (Vol. 204). Elsevier Science Limited.

[2] Kwun, Y. C., Farid, G., Nazeer, W., Ullah, S., \& Kang, S. M. (2018). Generalized Riemann-Liouville $k$-Fractional Integrals Associated With Ostrowski Type Inequalities and Error Bounds of Hadamard Inequalities. IEEE Access, 6, 64946-64953.

[3] Chen, H., \& Katugampola, U. N. (2017). HermiteÜHadamard and HermiteÜHadamardÜFejér type inequalities for generalized fractional integrals. Journal of Mathematical Analysis and Applications, 446(2), 1274-1291. 
[4] Habib, S., Mubeen, S., \& Naeem, M. N. (2018). Chebyshev type integral inequalities for generalized k-fractional conformable integrals. Journal of Inequalities and Special Functions, 9, 53-65.

[5] Jarad, F., Ugurlu, E., Abdeljawad, T., \& Baleanu, D. (2017). On a new class of fractional operators. Advances in Difference Equations, 2017(1), 247.

[6] Khan, T. U., \& Khan, M. A. (2019). Generalized conformable fractional operators. Journal of Computational and Applied Mathematics, 346, 378-389.

[7] Mubeen, S., \& Habibullah, G. M. (2012). k-Fractional integrals and application. International Journal of Contemporary Mathematical Sciences, 7(2), 89-94.

[8] Sarikaya, M. Z., Dahmani, Z., Kiris, M. E., \& Ahmad, F. (2016). (k,s)-Riemann-Liouville fractional integral and applications. Hacettepe Journal of Mathematics and Statistics, 45(1), 77-89.

[9] Andrić, M., Farid, G., \& Pečarić, J. (2018). A further extension of Mittag-Leffler function. Fractional Calculus and Applied Analysis, 21(5), 1377-1395.

[10] Salim, T. O., \& Faraj, A. W. (2012). A generalization of Mittag-Leffler function and integral operator associated with fractional calculus. Journal of Fractional Calculus and Applications, 3(5), 1-13.

[11] Rahman, G., Baleanu, D., Qurashi, M. A., Purohit, S. D., Mubeen, S., \& Arshad, M. (2017). The extended Mittag-Leffler function via fractional calculus. Journal of Nonlinear Sciences and Applications, 10, 4244-4253.

[12] Srivastava, H. M., \& Tomovski, Ž. (2009). Fractional calculus with an integral operator containing a generalized MittagÜLeffler function in the kernel. Applied Mathematics and Computation, 211(1), 198-210.

[13] Prabhakar, T. R. (1971). A singular integral equation with a generalized Mittag-Leffler function in the kernel. Yokohama Mathematical Journal, 19, 7-15.

[14] Sarikaya, M. Z., \& Ertuğral, F. (2017). On the generalized Hermite-Hadamard inequalities. https:/ /www.researchgate.net/publication/321760443. 